Dariusz Bugajewski, Faculty of Mathematics and Computer Science, Adam Mickiewicz University, Matejki 48/49, 60-769 Poznań, Poland. e-mail:

ddbb@main.amu.edu.pl

\title{
ON CARATHÉODORY'S CONDITIONS REVISITED
}

\begin{abstract}
In [3] we proved that generalized Carathéodory's conditions (the so called $(\mathrm{G})$ conditions) introduced in [6], imply well-known general conditions (the so called (CL) conditions) which guarantee existence and some properties of solutions of the Cauchy problem, in the Carathéodory sense. In this paper we prove that these two conditions are equivalent.
\end{abstract}

\section{Introduction}

Consider the following Cauchy problem

$$
x^{\prime}(t)=f(t, x), \quad x\left(t_{0}\right)=x_{0},
$$

where $I=\left[t_{0}, t_{0}+a\right] \subset \mathbb{R}, a>0, B=\left\{x \in \mathbb{R}^{n}:\left\|x-x_{0}\right\| \leq b\right\}$ and $f: I \times B \rightarrow \mathbb{R}^{n}$ is a Carathéodory function, i.e., it satisfies the classical Carathéodory (abbreviated: (C)) conditions (see [4] and [2], [7]).

By a solution of (1) in the Carathéodory sense, defined on some interval $J \subset I$, we understand a function $x: J \rightarrow \mathbb{R}^{n}$ such that $x(t) \in B$ for $t \in J, x$ is absolutely continuous on $J$, and $x^{\prime}(t)=f(t, x(t))$ for a.e. $t \in J$; equivalently, $x(t) \in B$ for $t \in J$ and

$$
x(t)=x_{0}+\int_{t_{0}}^{t} f(s, x(s)) d s \quad \text { for every } \quad t \in J,
$$

where the sign " $\int_{t_{0}}^{t} "$ denotes the Lebesgue integral. It is well known that if $f$ satisfies the conditions (C), then there exists an interval $J \subset I$ such that the

Key Words: Carathéodory conditions, Carathéodory solution, initial value problem

Mathematical Reviews subject classification: 26B35, 34A12

Received by the editors July 2, 1999 
problem (1) has a solution in the Carathéodory sense, defined on $J$ (see [5], pp. 54-56).

In [6] Grande formulated the conditions $(\mathrm{G})$, which are more general than the conditions (C) and guarantee the existence of a solution of (1) in the Carathéodory sense, on some interval $J \subset I$. Recall that a function $f$ : $I \times B \rightarrow \mathbb{R}^{n}$ satisfies the conditions (G) if

(i) for every continuous function $h: I \rightarrow B$ the superposition $t \rightarrow f(t, h(t))$, $t \in I$, is measurable;

(ii) there exists a Lebesgue integrable function $m: I \rightarrow \mathbb{R}_{+}$such that $\|f(t, x)\| \leq m(t)$ for every $(t, x) \in I \times B$

(iii) there exists a sequence of functions $f_{k}: I \times B \rightarrow \mathbb{R}^{n}$ satisfying the conditions (C) with $\left\|f_{k}(t, x)\right\| \leq m(t)$ for $(t, x) \in I \times B, k \in \mathbb{N}$, and such that for every subsequence $\left(f_{n_{k}}\right)$, for every sequence of continuous functions $g_{n}: I \rightarrow B$ which converges uniformly on $I$ to a function $g$, and for every $t \in I$, there is a strictly increasing subsequence sequence $\left(n_{i}\right)$ of $\left(n_{k}\right)$ of positive integers such that

$$
\lim _{i \rightarrow \infty} \int_{t_{0}}^{t} f_{k_{n_{i}}}\left(s, g_{n_{i}}(s)\right) d s=\int_{t_{0}}^{t} f(s, g(s)) d s
$$

Denote by $C\left(I, \mathbb{R}^{n}\right)$ the space of all continuous functions $I \rightarrow \mathbb{R}^{n}$ with the topology of uniform convergence and let $\widetilde{B}=\left\{z \in C\left(I, \mathbb{R}^{n}\right):\left\|z(s)-x_{0}\right\| \leq\right.$ $b$ for every $s \in I\}$.

If we replace (iii) by the following condition

(iv) if $z_{n}, z \in \widetilde{B}$ (for $n \in \mathbb{N}$ ) and $z_{n} \rightarrow z$ uniformly on $I$, then

$$
\int_{t_{0}}^{t} f\left(s, z_{n}(s)\right) d s \rightarrow \int_{t_{0}}^{t} f(s, z(s)) d s \quad \text { as } n \rightarrow \infty, \text { for every } t \in I,
$$

then we say that a function $f$ satisfies the conditions (CL).

In [3] we proved that if $f$ satisfies the conditions $(G)$, then it satisfies the conditions (CL). The aim of this paper is to establish that these two conditions are equivalent. 


\section{Results}

Denote by L the class of all subsets of $I$ which are measurable in the Lebesgue sense and by $\mu$ - the Lebesgue normalized measure. Further, denote by $S=$ $S\left(I, \mathbb{R}^{n}, \mu\right)$ the set of all equivalence classes of $\mu$ - measurable functions $I \rightarrow \mathbb{R}^{n}$ with the metric $\rho(x, y)=[x-y]$, where

$$
[z]=\inf _{0<p<+\infty}\{p+\mu(\{s: s \in I,|z(s)|>p\})\}, \quad x, y, z \in S .
$$

It is well known that convergence $\rho\left(x_{n}, x\right) \rightarrow 0$ as $n \rightarrow \infty$ is equivalent to convergence of $x_{n}$ in measure $\mu$ to $x$, i.e.,

$$
\mu\left(\left\{s \in I:\left|x_{n}(s)-x(s)\right|>p\right\}\right) \rightarrow 0 \quad \text { as } n \rightarrow \infty, \text { for any } p>0
$$

and $S$ becomes a complete metric space with respect to the above metric. Let $\widetilde{B}_{\mu}=\{z \in S: z(s) \in B$ for a.e. $s \in I\}$.

First, we prove the following

Lemma 1. Suppose that $f: I \times B \rightarrow \mathbb{R}^{n}$ satisfies (CL) conditions. If $z_{n}, z \in$ $\widetilde{B}_{\mu}$ for $n \in \mathbb{N}$ and $z_{n} \rightarrow z$ (in the sense of topology induced from $S$ ), as $n \rightarrow \infty$, then (2) is satisfied.

Proof. Let $z_{n}, z \in \widetilde{B}_{\mu}$ for $n \in \mathbb{N}$ and $z_{n} \rightarrow z$ as $n \rightarrow \infty$. Then, by Lusin's and Jegorov's theorems there exists a closed subset $D \subset I$ such that the restrictions $\left.z\right|_{D}$ and $\left.z_{n}\right|_{D}$ are continuous for $n \in \mathbb{N}$ and $\left.\left.z_{n}\right|_{D} \rightarrow z\right|_{D}$ uniformly on $D$. In view of Tietze's extension theorem there exist continuous functions $\tilde{z}_{n}, \tilde{z}$ such that $\left.\tilde{z}\right|_{D}=z$ and $\left.\tilde{z}_{n}\right|_{D}=z_{n}$ for $n \in \mathbb{N}$. Without loss of generality we may assume that $\tilde{z}, \tilde{z}_{n} \in \widetilde{B}$ for $n \in \mathbb{N}$ and $\tilde{z}_{n} \rightarrow \tilde{z}$ uniformly on $I$. In view of (iv) we have

$$
\int_{t_{0}}^{t} f\left(s, \tilde{z}_{n}(s)\right) d s \rightarrow \int_{t_{0}}^{t} f(s, \tilde{z}(s)) d s \quad \text { as } n \rightarrow \infty, \text { for every } t \in I,
$$

Fix $t \in I$ and $\varepsilon>0$. By the absolute continuity of the Lebesgue integral we can choose the above described set $D$ in such a way that $4 \int_{I \backslash D} m(s) d s<\frac{\varepsilon}{2}$.

In view of (3) there exists a positive integer $N$ such that

$$
\left|\int_{t_{0}}^{t}\left(f\left(s, \tilde{z}_{n}(s)\right)-f(s, \tilde{z}(s))\right) d s\right|<\frac{\varepsilon}{2} \text { for } n \geq N
$$


Hence, we obtain

$$
\begin{aligned}
& \left|\int_{t_{0}}^{t}\left(f\left(s, z_{n}(s)\right)-f(s, z(s))\right) d s\right| \leq \int_{t_{0}}^{t}\left|f\left(s, z_{n}(s)\right)-f\left(s, \tilde{z}_{n}(s)\right)\right| d s+ \\
& +\left|\int_{t_{0}}^{t}\left(f\left(s, \tilde{z}_{n}(s)\right)-f(s, \tilde{z}(s))\right) d s\right|+\int_{t_{0}}^{t}|f(s, \tilde{z}(s))-f(s, z(s))| d s< \\
& <2 \int_{I \backslash D} m(s) d s+\frac{\varepsilon}{2}+2 \int_{I \backslash D} m(s) d s<\varepsilon \text { for } n \geq N .
\end{aligned}
$$

Thus

$$
\lim _{n \rightarrow \infty} \int_{t_{0}}^{t} f\left(s, z_{n}(s)\right) d s=\int_{t_{0}}^{t} f(s, z(s)) d s
$$

which ends the proof of Lemma 1.

Corollary 1. Under the assumptions of Lemma 1 we have

$$
\int_{D} f\left(s, z_{n}(s)\right) d s \rightarrow \int_{D} f(s, z(s)) d s \quad \text { as } n \rightarrow \infty
$$

where $z, z_{n} \in \widetilde{B}_{\mu}$ for $n \in \mathbb{N}, z_{n} \rightarrow z$ as $n \rightarrow \infty$ and $D \in L$.

Proof. Denote by $\chi_{D}$ the characteristic function of a subset $D \in L$ and by $P_{D}$ the multiplication operator by $\chi_{D}$, i.e.,

$$
P_{D} x(s)=\chi_{D}(s) x(s), \quad s \in I,
$$

where $x: I \rightarrow \mathbb{R}$ is a $\mu$ - measurable function. Suppose for simplicity that $f(s, 0)=0$ for $s \in I$. Then we have

$$
f\left(s, P_{D} x(s)\right)=P_{D}(f(s, x(s))) \text { for } s \in I
$$

Let $z_{n} \rightarrow z$ as $n \rightarrow \infty$, where $z, z_{n} \in \widetilde{B}_{\mu}$ for $n \in \mathbb{N}$. Then $P_{D} z_{n} \rightarrow P_{D} z$ as $n \rightarrow \infty$. In view of Lemma 1 we obtain

$$
\int_{I} f\left(s, P_{D} z_{n}(s)\right) d s \rightarrow \int_{I} f\left(s, P_{D} z(s)\right) d s \quad \text { as } n \rightarrow \infty,
$$


so, by (4),

$$
\int_{I} P_{D}\left(f\left(s, z_{n}(s)\right)\right) d s \rightarrow \int_{I} P_{D}(f(s, z(s))) d s \quad \text { as } n \rightarrow \infty,
$$

and thus

$$
\int_{D} f\left(s, z_{n}(s)\right) d s \rightarrow \int_{D} f(s, z(s)) d s \quad \text { as } n \rightarrow \infty,
$$

where $D$ is any subset from $L$. The proof is completed.

In what follows we shall need the following result which is a version of Lemma 1.7 from [1, pp. 23-25]

Lemma 2. Assume that the function $f: I \times B \rightarrow \mathbb{R}^{n}$ satisfies the conditions (i), (ii) and

$$
\int_{D} f\left(s, z_{n}(s)\right) d s \rightarrow \int_{D} f(s, z(s)) d s \quad \text { as } n \rightarrow \infty,
$$

where $z, z_{n} \in \widetilde{B}_{\mu}$ for $n \in \mathbb{N}, z_{n} \rightarrow z$ as $n \rightarrow \infty$ and $D$ is any subset from $L$. Then there exists a sequence of functions $\left(f_{k}\right)$ such that every function $f_{k}: I \times B \rightarrow \mathbb{R}^{n}$ satisfies the conditions $(C)$ with $\left\|f_{k}(t, x)\right\| \leq m(t)$ for $(t, x) \in I \times B, k \in \mathbb{N}$ and

$$
\int_{D} f(s, z(s)) d s=\lim _{k \rightarrow \infty} \int_{D} f_{k}(s, z(s)) d s,
$$

for any subset $D$ from $L$.

Now, we are able to prove the following

Theorem 1. If $f: I \times B \rightarrow \mathbb{R}^{n}$ satisfies (CL), then it satisfies $(G)$.

Proof. Let $f: I \times B \rightarrow \mathbb{R}^{n}$ satisfy the conditions (CL). Then, by Corollary 1 and Lemma 2 there exists a sequence of functions $\left(f_{k}\right)$ such that every function $f_{k}: I \times B \rightarrow \mathbb{R}^{n}$ satisfies (C) conditions with $\left\|f_{k}(t, x)\right\| \leq m(t)$ for $(t, x) \in I \times B, k \in \mathbb{N}$ and

$$
\int_{t_{0}}^{t} f(s, z(s)) d s=\lim _{k \rightarrow \infty} \int_{t_{0}}^{t} f_{k}(s, z(s)) d s
$$


for every $z \in \widetilde{B}$ and $t \in I$. Fix $z \in \widetilde{B}$ and $t \in I$. Further, if $\left(z_{n}\right)$ is a sequence of functions from $\widetilde{B}$ such that $z_{n} \rightarrow z$ uniformly on $I$, as $n \rightarrow \infty$, then $f_{k}\left(\cdot, z_{n}(\cdot)\right) \rightarrow f_{k}(\cdot, z(\cdot)$ ) (in the sense of the topology of $S$ ) as $n \rightarrow \infty$, and thus

$$
\int_{t_{0}}^{t} f(s, z(s)) d s=\lim _{k \rightarrow \infty} \int_{t_{0}}^{t} \lim _{n \rightarrow \infty} f_{k}\left(s, z_{n}(s)\right) d s .
$$

In view of Vitali's theorem we obtain

$$
\int_{t_{0}}^{t} f(s, z(s)) d s=\lim _{k \rightarrow \infty}\left(\lim _{n \rightarrow \infty} \int_{t_{0}}^{t} f_{k}\left(s, z_{n}(s)\right) d s\right) .
$$

Consider a subsequence $\left(f_{k_{p}}\right)$. Fix $\varepsilon>1$. By (5) there exists a positive integer $k_{p_{1}}$ such that for every $k_{p} \geq k_{p_{1}}$ we have

$$
-1+\int_{t_{0}}^{t} f(s, z(s)) d s<\lim _{n \rightarrow \infty} \int_{t_{0}}^{t} f_{k_{p}}\left(s, z_{n}(s)\right) d s<1+\int_{t_{0}}^{t} f(s, z(s)) d s .
$$

Hence there exists a positive integer $n_{1} \geq p_{1}$ such that

$$
-1+\int_{t_{0}}^{t} f(s, z(s)) d s<\int_{t_{0}}^{t} f_{k_{n_{1}}}\left(s, z_{n_{1}}(s)\right) d s<1+\int_{t_{0}}^{t} f(s, z(s)) d s .
$$

Analogously, putting $\varepsilon=\frac{1}{2}$ we infer that there exists a positive integer $p_{2}>n_{1}$ such that

$$
-\frac{1}{2}+\int_{t_{0}}^{t} f(s, z(s)) d s<\lim _{n \rightarrow \infty} \int_{t_{0}}^{t} f_{k_{p}}\left(s, z_{n}(s)\right) d s<\frac{1}{2}+\int_{t_{0}}^{t} f(s, z(s)) d s .
$$

Hence there exists a positive integer $n_{2} \geq p_{2}$ such that

$$
-\frac{1}{2}+\int_{t_{0}}^{t} f(s, z(s)) d s<\int_{t_{0}}^{t} f_{k_{n_{2}}}\left(s, z_{n_{2}}(s)\right) d s<\frac{1}{2}+\int_{t_{0}}^{t} f(s, z(s)) d s .
$$

In this way putting $\varepsilon=\frac{1}{i}, i \in \mathbb{N}$ we obtain a subsequence $\left(f_{k_{n_{i}}}\right)$ with the property

$$
-\frac{1}{i}+\int_{t_{0}}^{t} f(s, z(s)) d s<\int_{t_{0}}^{t} f_{k_{n_{i}}}\left(s, z_{n_{i}}(s)\right) d s<\frac{1}{i}+\int_{t_{0}}^{t} f(s, z(s)) d s .
$$


Thus

$$
\lim _{i \rightarrow \infty} \int_{t_{0}}^{t} f_{k_{n_{i}}}\left(s, z_{n_{i}}(s)\right) d s=\int_{t_{0}}^{t} f(s, z(s)) d s
$$

which ends the proof.

It remains to state the last result of this paper which is an immediate consequence of Theorem 2.1 from [3] and Theorem 1, namely:

Theorem 2. The conditions $(G)$ and $(C L)$ are equivalent.

\section{References}

[1] J. Appel and P. Zabrejko, Nonlinear superposition operators, Cambridge University Press, 1990.

[2] G. Aquaro, Sul teorema di esistenza di Carathéodory per i sistemi di equazioni differenziali ordinarie, Boll. Unione Math. Ital. 8(1955), 208-212.

[3] D. Bugajewski and D. Wójtowicz, On generalized Carathéodory's conditions in ordinary differential equations, Math. Nachr. 199(1999), $71-75$.

[4] C. Carathéodory, Vorlesungen über reelle functionen, B. G. Teubner, Berlin, Leipzig, 1918.

[5] E. A. Coddington and N. Levinson, Theory of ordinary differential equations, McGraw Hill, New York, 1955.

[6] Z. Grande, On an integral equation, Math. Pann. 4(1)(1993), 95-101.

[7] Z. Opial, Sur l'équation différentielle ordinaire du premier ordre dont le second membre satisfait aux conditions de Carathéodory, Ann. Polon. Math. 8(1960), 23-28. 
